\title{
Photobase Generator Assisted Pitch Division
}

\author{
Xinyu $\mathrm{Gu}^{1}$, Christopher M. Bates ${ }^{2}$, Younjin $\mathrm{Cho}^{2}$, Takanori Kawakami ${ }^{1}$, Tomoki Nagai ${ }^{1}$, \\ Toshiyuki Ogata $^{2}$, Arun K. Sundaresan ${ }^{3}$, Nicholas J. Turro ${ }^{3}$, Robert Bristol ${ }^{4}$, Paul Zimmerman ${ }^{5}$, \\ C. Grant Willson ${ }^{1,2}$ \\ ${ }^{1}$ Department of Chemical Engineering and ${ }^{2}$ Department of Chemistry, The University of Texas at Austin, \\ Austin, TX 78712, USA; \\ ${ }^{3}$ Department of Chemistry, Columbia University, New York, NY 10027, USA; \\ ${ }^{4}$ Intel Corporation, TD, Hillsboro, OR 97124, USA; \\ ${ }^{5}$ Intel Assignee to SEMATECH, Austin, TX 78741, USA
}

\begin{abstract}
The drive to sustain the improvements in productivity that derive from following Moore's law has led the semiconductor industry to explore new technologies that enable production of smaller and smaller features on semiconductor device. Pitch division techniques and double exposure lithography are approaches that print features beyond the fundamental resolution limit of state-of-art lenses by modifying the lithographic process. This paper presents a new technique that enables pitch division in the printing of gratings using only a single exposure that is fully compatible with the current manufacturing tools. This technique employs a classical photoresist polymer together with a photoactive system that incorporates both a photoacid generator (PAG) and a photobase generator (PBG). The PBG is added to the resist formulation in higher molar concentration than the PAG, but has a base production rate that is slower than the acid production rate of the PAG. The PBG functions as a dose-dependent base quencher, which neutralizes the acid in high dose exposure regions but not in the low dose regions. This photoactive system can be exploited in the design of both positive tone and negative tone resist formulations that provide a developed image of a grating that is twice the frequency of the grating on the mask. A simulation of this process was performed for a $52 \mathrm{~nm}$ line and space pattern using PROLITH and customized codes. The results showed generation of a $26 \mathrm{~nm}$ half pitch relief image after development. Through this new technique, a $45 \mathrm{~nm}$ half pitch line and space pattern was experimentally achieved with a mask that produces a $90 \mathrm{~nm}$ half pitch aerial image. This corresponds to a $\mathrm{k}_{1}$ factor of 0.13 . The principles, the materials design and the first lithographic evaluations of this system are reported.
\end{abstract}

Keywords: pitch division, photobase generator, selective acid quenching, frequency doubling

Advances in Resist Materials and Processing Technology XXVII, edited by Robert D. Allen, Mark H. Somervell, Proc. of SPIE Vol. 7639, 763906 · @ 2010 SPIE · CCC code: 0277-786X/10/\$18 · doi: 10.1117/12.846395 


\section{INTRODUCTION}

\subsection{Motivation}

Following Moore's Law is becoming increasingly difficult and expensive. The resolution of optical exposure tools has reached its limit. Many techniques were proposed to extend the resolution limit of the optical exposure tools, including $157 \mathrm{~nm}$ lithography, immersion lithography with materials that have a higher refractive index than water, and so on. All of these approaches have now been abandoned. Double patterning is therefore the plan of record for many manufacturers. There are numerous variations on the theme of double exposure, but all require many more processing steps than have ever been used to produce the final image from photolithography. These processes work well, but the cost of ownership is high [1].

Pitch division is another approach to provide the desperately needed enhancement the resolution of $193 \mathrm{~nm}$ exposure tools. This technique requires only a single exposure and splits the pitch of aerial images in resist using extra processing steps. In this way, self alignment is achieved and the number of process steps is reduced. Excellent examples of pitch division techniques that are self-aligned double patterning (SADP) and dual-tone development (DTD). Both approaches have successfully demonstrated pitch division [2-4]. SADP demonstrated sub-32 nm half pitch line patterns with excellent LER/CD control. However, this approach requires many extra processing steps outside the exposure tool, which increases processing cost [3].

A still cheap pitch division technique, based on a dual-tone "hybrid" photoresist, was reported by IBM in 2000 [5]. This technique depends on a positive tone photoresist blended with a negative tone photoresist to form a "hybrid" resist, which demonstrates the dual-tone property. The unexposed resist is insoluble because an insufficient amount of acid is generated and the heavily exposed resist is also insoluble due to crosslinking by an excessive amount of acid, but areas of the resist that receive an intermediate dose are soluble. This solubility response occurs twice as frequent as the maximal intensity of the aerial image, thereby producing pitch division as shown in Fig. 1. Recently, a system based on the similar concept was described by MIT Lincoln Labs [6]. Both of these systems are impressive demonstrations of creativity and they achieved pitch division from a single exposure.

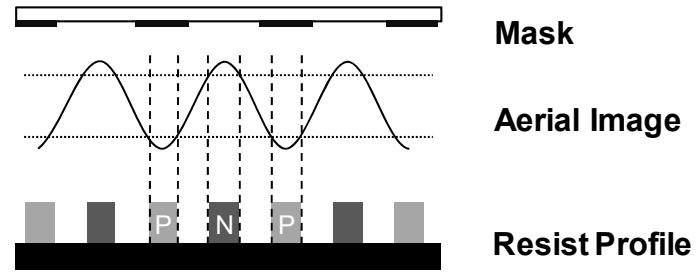

Fig. 1 Pitch division by a dual-tone "hybrid" photoresist composition from IBM [5]. At high dose region, the resist behaves as negative tone that is marked as ' $N$ '. At low dose region, the resist behaves as positive tone that is marked as ' $\mathrm{P}$ '.

In this paper, we report a new pitch division technique that requires exactly same steps as those in conventional lithography and is therefore fully compatible with the current manufacturing tools and processes. The only modification required to achieve pitch division is the addition of a component to the resist formulation. The doped resist has the capability of producing solubility responses at twice as the frequency of the aerial image, thereby doubling the frequency 
of a grating on the mask. The principle, simulation and experimental verification are presented in the following sections.

\subsection{Principle}

Pitch division can be accomplished if it is possible to create a resist response that has twice the frequency of the aerial image. To achieve pitch division, the kinetics of acid production in the resist, plotted as a function of dose, must be a parabola like curve as shown in Fig. 2. The dose-dependent acid concentration is low in both the lightly and heavily exposed areas, whereas the acid concentration reaches a maximum in the intermediate dose region. If the dissolution response threshold is below the maximal acid concentration in this plot, the development after post exposure bake (PEB) would produce the contrast curve at the bottom of Fig. 2 and would result in a lithographic pattern analogous to that is shown in Fig. 1.

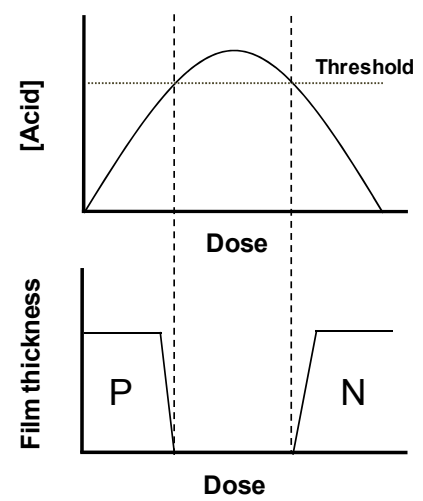

Fig. 2 A parabola like acid production curve of the sort that produces a dual tone response.

We have learned how to generate an acid production as a function of dose response like that shown in Fig. 2. The key component that allows production of this unusual parabolic acid profile is a photobase generator (PBG), a photoactive compound that generates bases upon UV irradiation. The photokinetics of base generation are similar to that of acid generation from the photoacid generator (PAG); both are first order growth. The photogenerated base and acid are produced simultaneously and neutralize each other. Thus, by adding a PBG to the resist formulation, the effective acid generation from PAG is no longer a typical first order growth curve. In the case where the rate constant for acid generation $\left(k_{a}\right)$ is greater than the rate constant for base generation $\left(k_{b}\right)$ and the initial PBG loading $\left([\mathrm{PBG}]_{0}\right)$ is greater than the initial PAG loading $[1]_{0}$, the photokinetics of base and acid generation can be described in the plot shown in Fig. 3 (a). The acid-base neutralization after exposure yields an inert salt and a net amount of either acid or base depending on the exposure dose. The highlighted area on the plot in Fig. 3 (a) shows the area where there is a net amount of acid after neutralization. This net acid can be directly plotted against dose, resulting in a parabola like curve as shown in Fig. 3 (b). The shape of this parabolic function is dependent on the relative PAG and PBG loadings and the rate constants of both base and acid generations. The influence of these factors on the chemical contrast was simulated and the results were reported earlier [7]. 
(a)

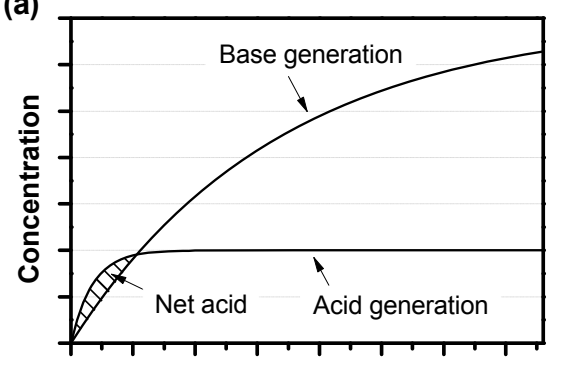

Dose (b)

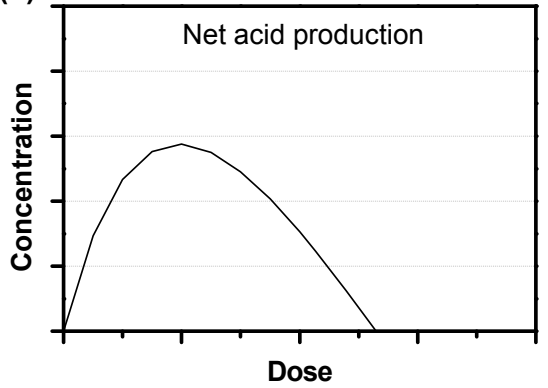

Fig. 3 (a) The photokinetics of an acid generation from a PAG and a base generation from a PBG in the case where $\mathrm{k}_{\mathrm{b}}<\mathrm{k}_{\mathrm{a}}$ and $[\mathrm{PBG}]_{0}>[\mathrm{PAG}]_{0}$. The net amount of acid is represented by the patterned area. (b) The net acid production against the exposure dose is a parabola like curve.

As described above, the parabola like acid production curve will enable a dual tone response and can lead to pitch division. While demonstration of a dual-tone contrast curve is necessary, it is not sufficient to produce pitch division. To enable pitch division, the contrast of the aerial image needs to be high enough to capture both the positive tone dose threshold $\left(\mathrm{E}_{0}\right)$ and the negative tone dose threshold $\left(\mathrm{E}_{\mathrm{n}}\right)$ in the resist. The match between the aerial image contrast and the dual-tone dose thresholds is therefore crucial to pitch division.

\section{SIMULATION}

\subsection{Analysis}

Pitch division requires a nearly sinusoidal aerial image, exactly the sort that is produced a the resolution limit of projection exposure tools. It also requires matching of the sinusoidal aerial image to the resist response. To better understand the necessity of matching the aerial image contrast with the dual-tone dose thresholds, one may first consider an aerial image with fixed contrast. When exposed with this aerial image, resist formulations with different values of $\mathrm{E}_{0}$ and $E_{n}$ yield different patterns as illustrated in Fig. 4. In all the cases, the exposure time was optimized to center the aerial image between the $E_{0}$ and $E_{n}$ in order to symmetrically capture the dose thresholds. Fig. 4 (a) shows the case in which the aerial image fails to capture both the positive and negative tone thresholds and Fig. 4 (b) shows the critical case in which the aerial image just captures both of the positive tone and the negative tone thresholds, producing a set of line patterns that are infinitesimal small. Clearly, variations in the exposure time can cause missing of the positive tone or the negative tone lines. In the case of Fig. 4 (c), the two thresholds are closer, which results in capturing of the aerial image at both high dose and low dose ends, producing wider lines. This graphical analysis shows how $\mathrm{E}_{0}$ and $\mathrm{E}_{\mathrm{n}}$ values in conjunction with the aerial image contrast determine the process window for pitch division and CD control. 
(a)

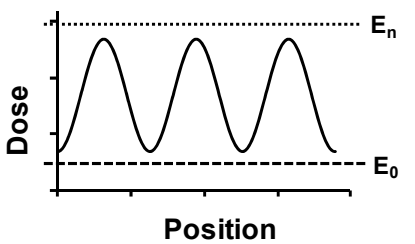

(b)

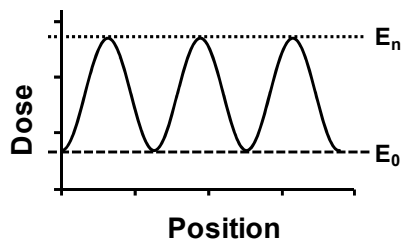

(c)

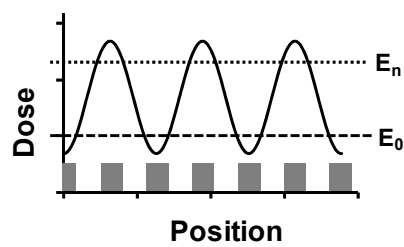

Fig. 4 The resist responses upon different matching of the aerial image contrast and the dose thresholds

It is important to clarify the definition of the aerial image contrast and the method used to evaluate the $E_{0}$ and $E_{n}$ values. The contrast of the aerial image is usually defined by Equation (1). The dual-tone dose thresholds $\left(\mathrm{E}_{0}\right.$ and $\left.\mathrm{E}_{\mathrm{n}}\right)$ can be bundled into a resist response parameter, the E-factor, which is defined in a fashion similar to that for the aerial image contrast as shown in Equation (2). Both the aerial image contrast and the E-factor are unitless.

$$
\begin{aligned}
& \text { Aerial image contrast }=\frac{I_{\max }-I_{\min }}{I_{\max }+I_{\min }} \\
& \text { E factor }=\frac{E_{n}-E_{0}}{E_{n}+E_{0}}
\end{aligned}
$$

The critical dimension (CD) error, the deviation from 1:1 L/S pattern is used to characterize the pitch division performance. For each combination of aerial image contrast and E-factor within the specific ranges, the CD of the resist pattern is determined by a simple calculation of the width of the captured aerial image at the dose thresholds. The corresponding CD errors, representing the influence of the aerial image contrast and the E-factor on the pitch division performance, are plotted in Fig. 5. This contour plot depicts how much the resist pattern deviates from a 1:1 L/S pattern. The black region, which stands for $100 \% \mathrm{CD}$ error, indicates no pitch division. The process window with 10\% CD error tolerance is indicated by the white area between two dashed lines on the plot and the dashed and dotted line in the center of this region shows 1:1 L/S condition. For a given aerial image contrast, a suitable range of E-factors can be found from the process window plot. In these calculations, the absorbance of the film was assumed negligible. 


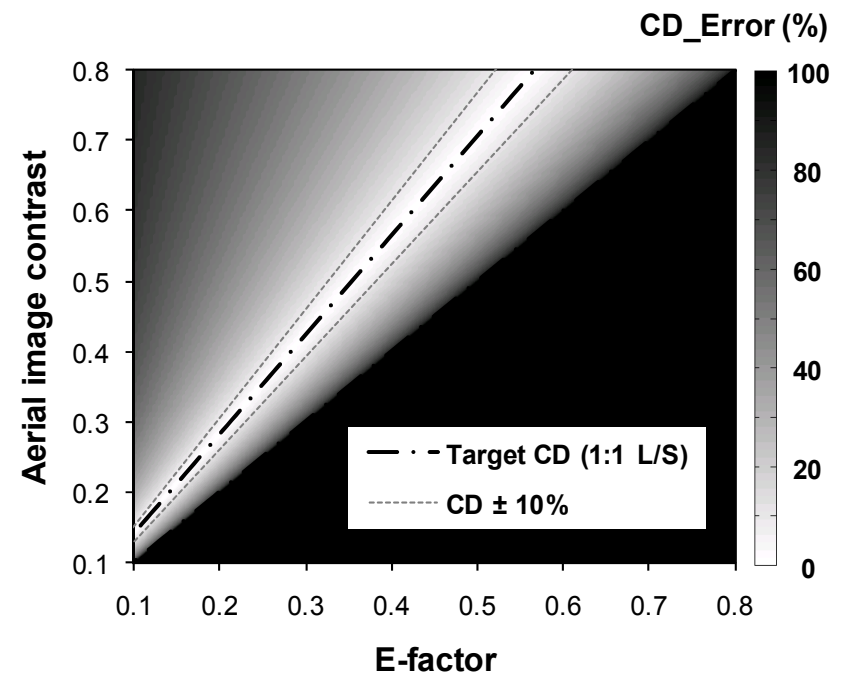

Fig. 5 The error of the feature $\mathrm{CD}$ is determined by the aerial image contrast and the E-factor.

\subsection{Simulation Conditions}

To test the lithographic feasibility of this approach, the resist profile and the process window were simulated in PROLITH 9.3.6 by employing a customized code that captures the fact that a non-conventional acid generation process is involved in this resist system. The photoactive system was simulated using a generic ArF resist to ensure applicability. The PEB temperature was optimized to obtain a proper E-factor for the target CD. A half pitch of $26 \mathrm{~nm}$ was targeted using a 1.1 NA water immersion exposure system. This is an effective $\mathrm{k}_{1}$ of 0.148 .

The major parameters involved in the lithography simulation are listed below. The illumination source was an azimuthally polarized dipole with $\sigma_{\text {center }}=0.8$ and $\sigma_{\text {radius }}=0.1$. The mask was $6 \%$ attenuated phase-shift mask with $52 \mathrm{~nm}$ $\mathrm{L} / \mathrm{S}$ pattern. The film stack was a $52 \mathrm{~nm}$ generic ArF resist $\left(\mathrm{n}=1.736, \mathrm{k}=0.028, \alpha=1.81 \mu \mathrm{m}^{-1}\right)$ on a $91.4 \mathrm{~nm}$ bottom anti-reflective coating $(\mathrm{BARC})(\mathrm{n}=1.82, \mathrm{k}=0.34)$. The parameters of the resist formulation are given in Table 1 .

Table 1 Crucial parameters of the processing condition

\begin{tabular}{c|c}
\hline Parameters (Unit) & Value \\
\hline PAG Loading (Equivalent) & 1.3 \\
{$[\mathrm{PBG}]_{0} /[\mathrm{PAG}]_{0}$} & 8 \\
$\mathrm{k}_{\mathrm{a}}\left(\mathrm{cm}^{2} / \mathrm{mJ}\right)$ & 0.0770 \\
$\mathrm{k}_{\mathrm{b}}\left(\mathrm{cm}^{2} / \mathrm{mJ}\right)$ & 0.00385 \\
{$[\mathrm{BQ}]($ Equivalent $)$} & 0 \\
$\mathrm{D}_{\text {acid }} @ 127^{\circ} \mathrm{C}\left(\mathrm{nm}{ }^{2} / \mathrm{sec}\right)$ & 2.61 \\
\hline
\end{tabular}

\subsection{Results}

The simulation of this approach yields a resist profile with pitch division. A $26 \mathrm{~nm}$ half pitch $\mathrm{L} / \mathrm{S}$ pattern was 
obtained from PROLITH as shown in Fig. 6.

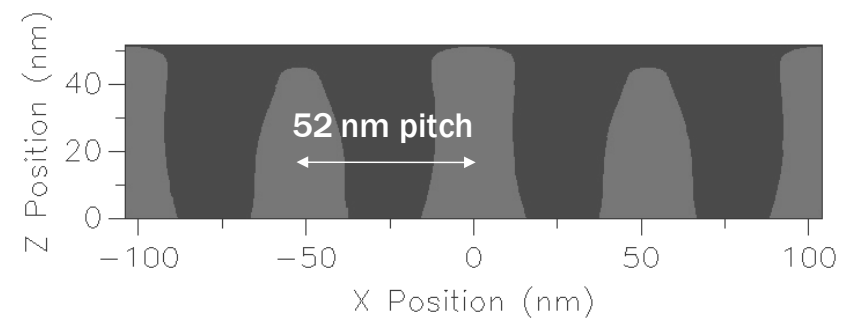

Fig. 6 The simulation result: $26 \mathrm{~nm} \mathrm{hp} \mathrm{L/S} \mathrm{pattern} \mathrm{on} \mathrm{the} \mathrm{wafer} \mathrm{through} \mathrm{a} \mathrm{mask} \mathrm{that} \mathrm{produces} \mathrm{an} \mathrm{aerial} \mathrm{image}$ with $52 \mathrm{~nm} \mathrm{hp} \mathrm{L/S}$

\section{EXPERIMENTAL SECTION}

\subsection{Photoactive system}

\subsubsection{Design of resist composition}

A formulation was created based on a PAG, a PBG and a generic $193 \mathrm{~nm}$ resin (MAdMA-co-GBLMA). Triphenolsulfonium perfluoro-n-butanesulfonate (TPS-Nf) was chosen as PAG. Extensive studies have been done on PBGs $[8,9]$ from which $o$-nitrobenzyl carbamate type compounds were chosen for the initial test. The PBGs were synthesized according to the published procedures [7, 9-12]. The mechanism of the photolysis that produces base in this class of compounds is well characterized [13]. The PBG candidates that were synthesized are shown in Fig. 7.<smiles>CC(C)N(C(=O)OCc1ccccc1[N+](=O)[O-])C(C)C</smiles>

DIPA-CARB

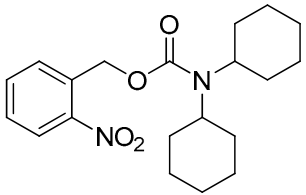

DCHA-CARB

Fig. 7 The structures of PBGs: $o$-nitrobenzyl N,N-diisopropylcarbamate (DIPA-CARB) and o-nitrobenzyl

N,N-dicyclohexylcarbamate (DCHA-CARB)

\subsubsection{Materials evaluation}

The sample solutions were formulated in the following way. The resin was first dissolved into 2-heptanone to form a $5 \mathrm{wt} \%$ mother solution. The masses of TPS-Nf and DCHA-CARB were calculated based on $6.5 \mathrm{wt} \%$ PAG loading with respect to the resin and 3:1 molar ratio of PBG to PAG. The calculated amount of photoactive compounds was then added to this mother solution to formulate the final sample solution. The resin loading, the PAG loading and the PBG/PAG feed ratio are the three factors that determine the relative concentration of each component in the solution. The kinetics of both acid and base generation must be also considered in the sample preparation to ensure a parabola like net acid production curve. From their rate constants, an appropriate range of the PBG/PAG feed ratio can be predicted to facilitate the formulation. The relative measurements and analysis were reported in the previously published paper [7]. 
Fig. 8 shows the contrast curve of the above sample formulation at a certain condition $(120 \mathrm{~nm}$ film thickness, PAB @ $110^{\circ} \mathrm{C}$ for $60 \mathrm{sec}$, PEB @ $121.5^{\circ} \mathrm{C}$ for $60 \mathrm{sec}$, develop in TMAH for $60 \mathrm{sec}$ ). The dual-tone behavior can be easily observed in this plot. The critical threshold dose was defined as the dose that leads to half of the original film thickness after development. The $\mathrm{E}_{0}$ and the $\mathrm{E}_{\mathrm{n}}$, representing the positive tone and the negative tone dose thresholds, are indicated in the plot as defined.

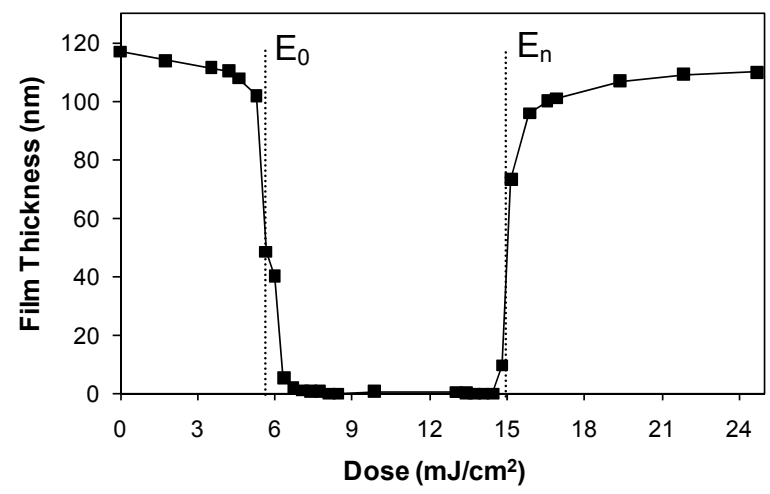

Fig. 8 The contrast curve for a sample formulation consisting of $6.5 \mathrm{wt} \%$ TPS-Nf (with respect to the resin) and 3 times DCHA-CARB of TPS-Nf loading in moles.

Although the slope of each tone depicts the contrast of the dissolution modulation to some extent, a better description of this contrast would be a plot of dissolution rate against dose. For the proof-of-concept, the slopes of the contrast curve were not considered as a factor to the $C D$ control. Only the $E_{0}$ and $E_{n}$ values were derived from the contrast curve plot.

\subsection{Optimization}

Intuitively, the $\mathrm{CD}$ can be adjusted by tuning the aerial image contrast or the E-factor. The higher the contrast of the sinusoidal aerial image, the sharper the resist pattern at the wafer plane. The E-factor can be readily adjusted to match the aerial image contrast because it is dependent on the PEB temperature, the PAG loading, the PAG to PBG feed ratio, the developing time variables and so on, all of which are easy to adjust. The first three parameters affect the E-factor most strongly. An optimization of these three parameters is needed to find the appropriate range of the E-factor that would produce 1:1 L/S pattern with a specified tolerance of $\mathrm{CD}$ error for a given aerial image contrast.

The Box-Behnken design was employed as an experimental method to find the best combination of the three processing parameters for the target value of the E-factor. The Box-Behnken design is an independent quadratic design that is used to establish the interaction between three orthogonal parameters and produce a continuous response surface. 


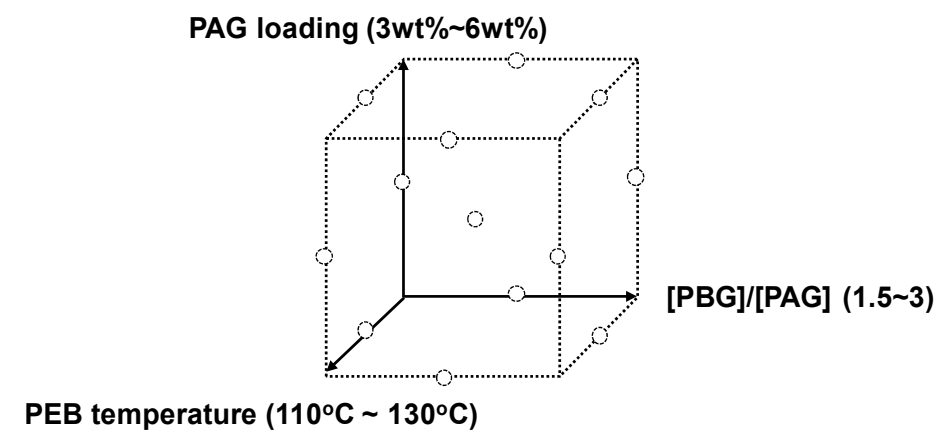

Fig. 9 Box-Behnken design for optmization of processing parameters

The three parameters are independent of each other and respectively assigned for three axes in a 3-D coordinate. The $E_{0}$ and $E_{n}$ were determined experimentally at three levels of each factor, indicated as circles in Fig. 9. According to the Box-Behnken design, this combination of experiments can predict the response surface $[14,15]$ with a reasonable accuracy.
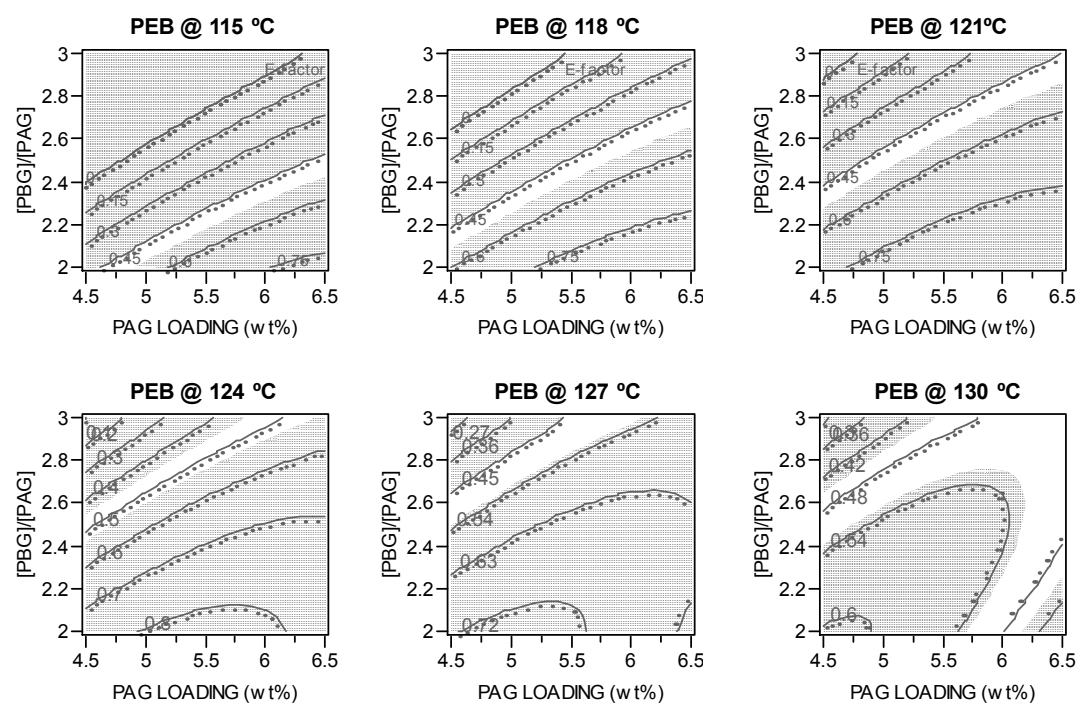

Fig. 10 The contour plots of the response surface at various PEB temperatures

The contour plot in Fig. 10 presents the response surface of the E-factor for one of the PBGs, DCHA-CARB. The white regions on the plot are the process window of the E-factor that produces 1:1 L/S patterns within 10\% CD error. The specified range of the E-factor was suggested by Fig. 5 assuming a certain aerial image contrast from the exposure tool. These contour plots can be used as a guide for process parameter settings to achieve pitch division.

\subsection{Lithography results}

The dual tone resist system was exposed on an interferometric lithography tool, which is called Amphibian ${ }^{\mathrm{TM}}$ system developed at RIT [16]. The 0 order diffraction pattern is stopped by a beam blocker so that only the -1 and +1 order are captured, producing a nearly perfect sinusoidal aerial image.

The resist formulations were prepared in the manner as described in the experimental section. A particular sample 
formulation (4.14wt \% TPS-Nf and 2.5 equ of DCHA-CARB to TPS-Nf in MAdMA-co-GBLMA) was exposed with the interferometric lithography tool at $\mathrm{NA}=0.22$, that produces an aerial image with $220 \mathrm{~nm}$ half pitch. After the post exposure processes (PEB @ $115^{\circ} \mathrm{C}$ for $60 \mathrm{sec}$, develop in 2.38\% TMAH for $60 \mathrm{sec}$ ), the resist image revealed a $110 \mathrm{~nm}$ $\mathrm{hp} \mathrm{L/S} \mathrm{pattern} \mathrm{with} \mathrm{pitch} \mathrm{division} \mathrm{as} \mathrm{shown} \mathrm{in} \mathrm{Fig.} \mathrm{11.} \mathrm{This} \mathrm{is} \mathrm{an} \mathrm{effective} \mathrm{k}_{1}$ of 0.15 which is lower than the resolution limit for a lens with that numerical aperture at $193 \mathrm{~nm}$ exposure wavelength.
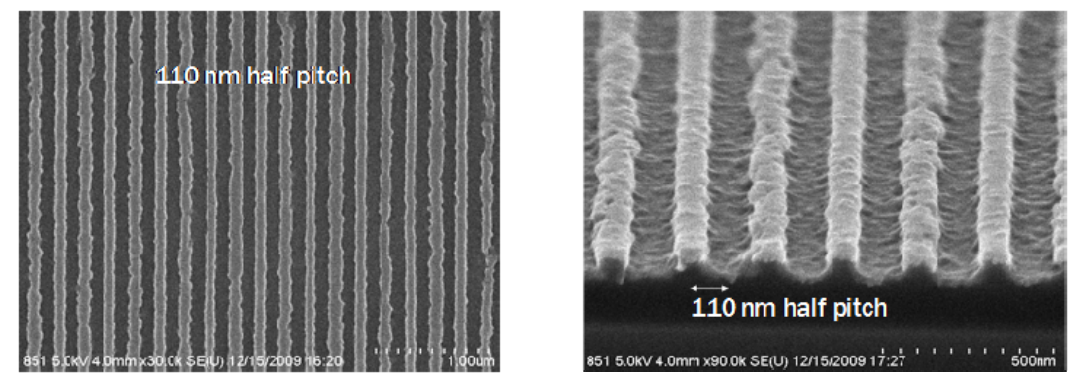

Fig. 11 The SEM images of resist pattern $(110 \mathrm{~nm} \mathrm{hp})$ created on an interferometric lithography tool $(\mathrm{NA}=0.22)$ using a dual-tone resist formulation.

Smaller feature size requires aerial images with tighter pitches, which can be achieved by increasing the NA of the exposure tool. An aerial image with $90 \mathrm{~nm}$ half pitch can be obtained at NA $=0.54$. However, there was no success for pitch division with MAdMA-co-GBLMA at that pitch size. The exact reason is unclear, but the generic polymer employed is not optimized for high resolution.

A quick method for testing this pitch division approach at smaller dimension is to add a PBG into a commercial ArF resist, which was already optimized for sub-32nm imaging. Two commercial ArF resists from leading resist vendors were tested with PBGs and both exhibited dual tone behavior. The result shown in Fig. 12 is a $45 \mathrm{~nm} \mathrm{hp} \mathrm{L/S} \mathrm{pattern} \mathrm{that}$ was produced by a $90 \mathrm{~nm} \mathrm{hp}$ aerial image at $\mathrm{NA}=0.54$. This is a result of pitch division and an effective $\mathrm{k}_{1}$ of 0.13 . Although the LER is fairly large, this result is still a satisfying proof-of-concept.

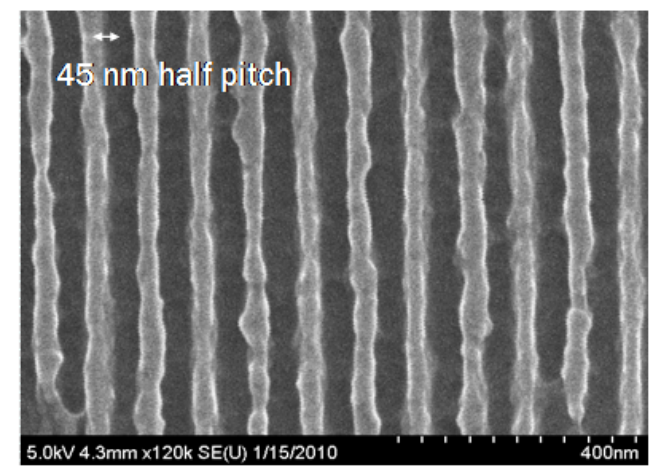

Fig. 12 An SEM image of the resist pattern $(45 \mathrm{~nm} \mathrm{hp})$ created on an interferometric lithography tool (NA $=0.54,90 \mathrm{~nm}$ hp aerial iamge) with a resist formulation by adding the DCHA-CARB into a commercial ArF resist

\section{CONCLUSION}


A simple pitch division process is reported that has an acceptable process latitude. Pitch division is achieved by selective acid quenching using photobase generators to create a resist profile with both negative tone and positive tone lines, dividing the pitch on the mask by a factor of 2 . The required materials were successfully synthesized and demonstrated in a test system. The formulations and the processing conditions were adjusted to give 1:1 lines and spaces by both simulation and experiments. The lithographic results show pitch division at $110 \mathrm{~nm} \mathrm{hp}$ and $45 \mathrm{~nm}$ hp, providing a satisfying proof of concept. Continued effort will be applied toward reduction of the LER and auditioning of new photobase generators.

\section{ACKNOWLEDGEMENT}

The authors would like to thank SEMATECH Engineering Research Center, Intel and The Rushed Engineering Regent's Chair for funding. SEMATECH and the SEMATECH logo are registered service marks of SEMATECH, Inc. All other service marks and trademarks are the property of their respective owners.

\section{REFERENCE}

[1] R.H. French, and H.V. Tran, "Immersion Lithography: Photomask and Wafer-Level Materials," Annual Review of Materials Research 39(1), 93-126 (2009).

[2] C. Fonseca, M. Somervell, S. Scheer, W. Printz, K. Nafus, S. Hatakeyama, Y. Kuwahara, T. Niwa, S. Bernard, and R. Gronheid, "Advances and challenges in dual-tone development process optimization," Proc. of SPIE 7274, 72740I-72712 (2009).

[3] C. Bencher, Y. Chen, H. Dai, W. Montgomery, and L. Huli, "22nm half-pitch patterning by CVD spacer self alignment double patterning (SADP)," Proc. of SPIE 6924, 69244E-69247 (2008).

[4] W. Shiu, H.J. Liu, J.S. Wu, T.-L. Tseng, C.T. Liao, C.M. Liao, J. Liu, and T. Wang, "Advanced self-aligned double patterning development for sub-30-nm DRAM manufacturing," Proc. of SPIE 7274, 72740E-72747 (2009).

[5] M.C. Hakey, S.J. Holmes, D.V. Horak, A.D. Katnani, N.M. Patel, and P.A. Rabidoux, "Frequency doubling hybrid photoresist having negative and positive tone components and method of preparing the same," US Patent, (2000).

[6] T.H. Fedynyshyn, SEMATECH Workshop on Novel Extensions of Optical, (2007).

[7] X. Gu, C. Bates, Y. Cho, E. Costner, F. Marzuka, T. Nagai, T. Ogata, C. Shi, A.K. Sundaresan, N.J. Turro, R. Bristol, P. Zimmerman, and C.G. Wilson, "A New Materials-based Pitch Division Technique," Journal of Photopolymer Science and Technology 22(6), 773-781 (2009).

[8] K. Suyama, S. Nakao, and M. Shirai, "Thermally stable carbamates as novel photobase generator," $J$. Photopolym. Sci. and Technol. 18(1), 141-148 (2005).

[9] K. Suyama, and M. Shirai, "Photobase generators: Recent progress and application trend in polymer systems," 
Progress in Polymer Science 34(2), 194-209 (2009).

[10] J.F. Cameron, and J.M.J. Frechet, "Photogeneration Of Organic-Bases From Ortho-Nitrobenzyl-Derived Carbamates," J. Am. Chem. Soc. 113(11), 4303-4313 (1991).

[11] H. Lange, R. Huenerbein, B. Wibbeling, R. Fröhlich, S. Grimme, and D. Hoppe, "Comprehensive Experimental and Theoretical Studies of Configurationally Labile Epimeric Diamine Complexes of alpha-Lithiated Benzyl Carbamates," Synthesis 2008(18), 2905-2918 (2008).

[12] S. Martina, S.A. MacDonald, and V. Enkelmann, "Photosensitive Tetramethylpiperidine Urethanes: Synthesis and Characterization," The Journal of Organic Chemistry 59(12), 3281-3283 (1994).

[13] F.M. Houlihan, A. Shugard, R. Gooden, and E. Reichmanis, "Nitrobenzyl Ester Chemistry For Polymer Processes Involving Chemical Amplification," Macromolecules 21(7), 2001-2006 (1988).

[14] G.E.P. Box, and D.W. Behnken, "Some New Three Level Designs for the Study of Quantitative Variables," Technometrics 2(4), 455-475 (1960).

[15] J. Sall, and A. Lehman, "JMP Start Statistics," Duxbury Press. 344-348 (1996).

[16] W.S. Bruce, B. Anatoly, F. Yongfa, C. Frank, and H. Peter, "Amphibian XIS: an immersion lithography microstepper platform," Proc. of SPIE 5754, 751-759 (2004). 